\title{
Occipital nerve block and Maitland physiotherapy: review of treatment techniques based on positive results
}

\author{
Wesley Gabriel Novaes Botelho (D), Laura Suelen Signori (D), Alcântara Ramos de Assis César \\ Universidade Federal do Parana, Parana, Brazil
}

\section{$凶$}

Wesley Gabriel Novaes Botelho wesleygabrielnovaesbotelho@ gmail.com

\section{Edited by:}

Marcelo Moraes Valença

Keywords:

Neck pain

Physical therapy modalities

Nerve block

\begin{abstract}
Cervicogenic headache is a clinical condition that has a direct impact on quality of life and labor productivity and its underdiagnosis and lack of consensus on the appropriate treatment aggravates even more the condition. This work aims to systematize the protocol that has the highest success rate so far, based on the study carried out in Pindamonhangaba, São Paulo, Brazil. The results demonstrated with the occipital block and Maitland physiotherapy were exceptional and its methodology will be carried out as performed during the study.

Conclusion: Regardless of the substance used for the blockade, its combination with Maitland's physiotherapy was shown to be favorable and its description can help other professionals and also enable reproduction in other studies.
\end{abstract}




\section{Introduction}

Headache is one of the most prevalent conditions in the - population, resulting in quality of life impairment and reduced labor productivity. Headaches can be divided into several groups, and these groups are classified as primary and secondary headaches, according to the International Headache Society. ' Symptomatic overlap leads to an underdiagnosis of cervicogenic headache, and $50 \%$ of cases have inadequate diagnosis and treatment. ${ }^{2}$

Cervicogenic headaches are classically included in a large group of secondary headaches associated with neck disorders because their symptomatology and etiology is characterized by a different pattern from migraine and tension headache, not being considered in an isolated category according to the International Headache Society. ' The pain has a variable intensity, usually moderate, lasting from a few hours to weeks, with a pattern of higher incidence from 1 to 3 days, not throbbing and not excruciating. It may present prodromes related to the C2-C3 nerve roots, such as throbbing and pinching in the neck and occipital region ipsilateral to pain, hypoesthesia, dysphagia, xerostomia, and stiffness in the passive movement of the neck. ${ }^{3}$

Currently, there are two diagnostic criteria panels, the one adapted by Antonaci and colleagues ${ }^{4}$ and the International Headache Society. ${ }^{1}$

Diagnostic criteria adapted by Antonaci and colleagues: ${ }^{4}$

1. Unilateral headache without lateral displacement.

2. Symptoms and signs of neck involvement: pain triggered by neck movement or inadequate sustained posture, and/ or external pressure on the posterior region of the occipital neck; ipsilateral neck or occiput pain; ipsilateral neck, shoulder, and arm pain; reduced range of motion.

3. Pain episodes with variable duration or continuous and fluctuating pain.

4. Moderate pain, not throbbing and not excruciating.

5. Pain starting in the neck, radiating to the orbital, frontal, and temporal regions.

6. Temporary relief of pain with anesthetic blocks provided that a complete anesthesia or sustained cervical trauma occurs shortly before onset.

7. Several events related to the attack: autonomic signs and symptoms, nausea, vomiting, ipsilateral edema and redness in the periocular area, dizziness, phonophobia, photophobia, or ipsilateral blurred vision.

For the diagnosis, it is necessary the presence of items 1 and 5, and any other three.

Diagnostic criteria of the International Headache Society 1:

A. Any headache that meets criterion $C$.

B. Clinical and/or imaging evidence of a cervical spine or neck soft tissue disorder or injury known to cause headache.

C. Evidence of causality demonstrated by at least two of the following findings:

1. The headache developed in a temporal relation with the cervical disorder or lesion onset

2. The headache significantly improved or resolved in parallel with the recovery or resolution of the cervical disorder or injury.

3. Cervical range of motion is reduced and headache is significantly aggravated by provocative maneuvers 4. Headache is abolished after diagnostic blockage of a cervical structure or its nerve supply

D. Not better explained by another diagnosis.

Notes:

1. High cervical spine imaging findings are common in patients without a headache; they are suggestive but not solid evidence of causality.

2. Upper cervical spine tumors, fractures, infections, and rheumatoid arthritis have not been formally validated as causes of headache, but are accepted to meet criterion B in individual cases. Cervical spondylosis and osteochondritis may or may not be valid causes that meet criterion B, again depending on the individual case.

3. When cervical myofascial pain is the cause, the headache should probably be classified as tensiontype headache; however, awaiting more evidence, an alternative diagnosis of headache attributed to cervical myofascial pain can be raised.

4. Headache caused by upper cervical radiculopathy has been postulated and, considering the now wellunderstood convergence between upper cervical and trigeminal nociception, this is a logical cause of headache. Depending on other evidence, this diagnosis has as a differential one the headache attributed to superior cervical radiculopathy.

5. Features that tend to distinguish cervicogenic from 
migraine and tension-type headache include sideways pain, typical headache provocation by digital pressure on neck muscles and head movement, and posterioranterior radiation of the pain. However, while these may be features of cervicogenic headache, they are not exclusive to it and do not necessarily define causal relation. Migraine features such as nausea, vomiting, and photo/phonophobia may be present in cervicogenic headaches, although to a generally lower degree than in migraine, and may differentiate some cases of tensiontype headaches.

Regarding the treatments, several methods and suggested pharmacology, all with limited success were found. ${ }^{5}$ Among the used drugs there are muscle relaxants, antidepressants, and neuromodulators. ${ }^{6}$ Non-pharmacological therapies are currently the most accepted treatment for cervicogenic headache, with effective and timely restricted remission. The isolated blockade had an immediate response in $94 \%$ of patients, however, the mean pain-free duration was 23.5 days. $^{7}$ Excision of the major occipital nerve and radiofrequency neurotomy were not shown as effective, even with the average of 244 days and 297 days with pain reduction, respectively, the studies are inconclusive. ${ }^{5}$ A study conducted in Brazil, with solid methodology and statistical significance, associated the Maitland technique with the occipital nerve block on a scale from 1 to 10), with a score of 1.6 for combined treatment with Maitland technique and block, 3.7 for conventional physical therapy combined with block and 5.2 for isolated block 6 . The goal of this paper is to systematize block and physical therapy techniques, based on work of CÉSAR and colleagues (2021), to better guide the effective treatment of cervicogenic headaches.

\section{Methodology}

The present study used the systematization of the techniques used by CÉSAR and colleagues (2021), in the study carried out at the 10 de Julho Hospital, in Pindamonhangaba, São Paulo, Brazil, from January 2017 to February 2018.

\section{Protocols}

The etiology of cervicogenic headache is secondary to several disorders that affect cervical and trigeminal afferent nerve roots in the trigemiocervical nucleus. In the nucleus, nociceptive afferents from spinal roots C1. C2-C3 converge to second-order neurons that also receive afferents from cervical nerves and from the first division of the trigeminal nerve. These convergences allow high neck pain to be referred in the domain regions of the cervical nerves (occipital and auricular) and also in the path and the region innervated by the first division of the trigeminal (parietal, frontal and orbicular). ${ }^{5}$

Considering the anatomical and drug features, we will describe the protocol:

1. Prepare material for sterilization, syringe, 2 needles, $1 \%$ lidocaine without vasoconstrictor, $2 \mathrm{mg}$ dexamethasone.

2. Aspirate $1 \mathrm{ml}$ of $2 \mathrm{mg}$ dexamethasone and $2 \mathrm{ml}$ of $1 \%$ lidocaine without vasoconstrictor.

3. Perform local antisepsis.

4. Identify the path of the major occipital nerve, which is located on the lateral third of the occipital protrusion in a line going from the occipital protrusion to the mastoid process.

5. Apply the substance subcutaneously with a 26 G 13 $\mathrm{mm}$ needle.

The effect is immediate, and a reassessment is recommended after 20 days. 8,9

Regarding the Maitland physiotherapy protocol, there are basic characteristics of the mobilization degree..$^{10,11}$

Grade I - small amplitude movement close to the starting position of the path;

Grade II - large amplitude movement within the path. It can take any part of the path that is free of any muscle stiffness or spasm;

Grade III - large amplitude movement, but within muscle stiffness or spasm, at the range end;

Grade IV - small range movement forced into muscle stiffness or spasm at the range end.

Grades I and II are indicated in the initial therapy to reduce pain and irritation, grades III and IV are used in more advanced therapies to increase the range of motion, stabilization, and stretching of the joint capsule.

The mobilization rate must be $2 \mathrm{~Hz}$ and the period of 30 to 60 seconds per manipulation.

The recommended physiotherapy period is 8 weeks.

These protocols are prepared by the authors of the study based on clinical practice and are supported by scientific evidence.

\section{Conclusion}

Based on previous studies, it is possible to conclude that, 
regardless of the substance used, the isolated blockade has a limited improvement in cervicogenic headache symptoms. ${ }^{6,7,12}$ The association of major occipital nerve block with simple physical therapy demonstrated more efficacy than the simple block and less than the block associated with the Maitland technique. ${ }^{6}$

The work concludes its goal of systematizing the technique used in the research, for protocol use by other professionals. It is important in the medical field to improve the diagnosis and treatment rates of cervicogenic headache. Furthermore, it enables the reproducibility of the method for conducting studies by other professionals in the field.

\section{Autor contributions}

Wesley Gabriel Novaes Botelho: First author, elaboration of protocols following the primary study, elaboration of the base text.

Laura Suelen Signori: Co-author, review of protocols and translation.

Alcântara Ramos de Assis César: Advisor.

\section{Conflict of interests}

This study is free from conflict of interest.

Financing

Own financing.

Wesley Gabriel Novaes Botelho

https://orcid.org/0000-0001-7315-4201

Laura Suelen Signori

https://orcid.org/0000-0002-9397-6605

Alcântara Ramos de Assis César

https://orcid.org/0000-0002-7390-7282

\section{References}

1. Headache Classification Committee of the International Headache Society (IHS) The International Classification of Headache Disorders, 3rd edition. Cephalalgia 2018;38(1):1-211 Doi: $10.1177 / 0333102417738202$

2. Anarte-Lazo E, Carvalho GF, Schwarz A, Luedtke K and Falla D. Differentiating migraine, cervicogenic headache and asymptomatic individuals based on physical examination findings: a systematic review and meta-analysis. BMC Musculoskelet Disord
2021;22(1):755 Doi:10.1186/s12891-021-04595-w

3. Correia $C$ and Monteiro P. Cefaleia cervicogénica. Acta Med Port 1992;5(3):155-158

4. Antonaci F, Ghirmai S, Bono G, Sandrini G and Nappi $G$. Cervicogenic headache: evaluation of the original diagnostic criteria. Cephalalgia 2001;21(5):573583 Doi:10.1046/i.0333-1024.2001.00207.x

5. Bogduk N and Govind J. Cervicogenic headache: an assessment of the evidence on clinical diagnosis, invasive tests, and treatment. Lancet Neurol 2009;8(10):959-968 Doi:10.1016/s14744422(09)70209-1

6. Assis César AR, Costa IDP, Botelho WGN, Sperafico $N$, Groto AD, Felício ACJR, Society and Development. Postural physiotherapy for cervicogenic headache after occipital nerve block: a retrospective study. Res Soc Dev. 2021;10(12):e165101219994 -e 165101219994 Doi:10.33448/rsd-v10i12.19994

7. Anthony M. Cervicogenic headache: prevalence and response to local steroid therapy. Clin Exp Rheumatol 2000; 18(2 Suppl 19):S59-64

8. Tepe $\mathrm{N}$ and Tertemiz OF. Comparison of greater occipital nerve and greater occipital nerve + supraorbital nerve block effect in chronic medication overuse headache. Turk J Med Sci 2021;51(3):1065. 1070 Doi:10.3906/sag-2009-101

9. Kim JS, Ko JS, Bang S, Kim H and Lee SY. Cervical plexus block. Korean J Anesthesiol 2018;71(4):274288 Doi:10.4097/kja.d. 18.00143

10. Karvat J, Antunes JS and Bertolini GRFJRD. Posteroanterior lumbar spine mobilizations in healthy female volunteers. Evaluation of pain to cold and pressure: crossover clinical trial. Rev Dor 2014;15(1):21-24 Doi:10.5935/18060013.20140006

11. Bailey E, Heneghan NR, Cassidy NJ, Falla D and Rushton $A B$. Clinical effectiveness of manipulation and mobilisation interventions for the treatment of nonspecific neck pain: protocol for a systematic review and meta-analysis. BMJ Open 2020;10(10):e037783 Doi:10.1136/bmiopen-2020-037783

12. Wang $E$ and Wang D. Treatment of cervicogenic headache with cervical epidural steroid injection. Curr Pain Headache Rep 2014;18(9):442 Doi:10.1007/ s1 1916-014-0442-3 